\title{
EER-AL: AN ENERGY EFFICIENT ROUTING PROTOCOL BASED ON AUTOMATED LEARNING METHOD
}

\author{
Farzad Kiani \\ Department of Computer Engineering, Engineering and Natural Sciences Faculty, Istanbul Sabahttin Zaim University, Turkey
}

\begin{abstract}
The issue of energy in a wireless sensor network is one of the most important challenges for these networks. This issue is also being considered today in the new IoT topic. This paper studies the ability of the learning automata model to solve the problem in the sensor networks. Because they have capabilities such as low computational load, ability to use in distributed environments, and inaccurate information, require the least feedback from the environment, etc. One of the solutions to energy optimization is to provide routing protocols. In the routing area, a routing protocol based on learning automata has been proposed in which the network lifetime criterion is considered. The simulation results and the comparison of the proposed protocol with other protocols indicate that this protocol has better performance in the energy conversation and network lifetime.
\end{abstract}

Keywords:

Wireless Sensor Networks, Energy Efficiency, Routing Protocol, Fault Tolerance, Automated Learning

\section{INTRODUCTION}

Wireless sensor network (WSN) is a special type of ad-hoc networks and includes a set of small cheap nodes that these nodes can sense the environment for a specific purpose, processing information, storing, exchanging information with other nodes, and the ability adoption against changes (topology, etc.). Usually all the nodes are identical and in practice, they work together to meet the overall purpose of the network. These nodes have some limitations such as battery and memory. Therefore, the efficient use of these resources is influential in the process of reaching the network goal. In recent days, the issue of Internet of Things (IoT) is a popular topic that it is from a family of WSNs. IoT, sometimes referred to as the Internet of Objects, will change everything including ourselves [1].

The main goals in these networks can be different, such as monitoring and controlling the conditions and changes in atmospheric, physical or chemical. Therefore, the network design is based on environment requirements. Many factors need to be considered in design as network topology, scalability, fault tolerance, data reporting etc. [2]. Each designed system, given its intrinsic properties, requires certain conditions and situations. When used in those situations and situations, it has some advantages and disadvantages to its similar systems. It should ultimately choose a system that has the best cost-effectiveness by an implicit estimate and according to all available conditions. The WSNs have some advantages such as quick setup in emergencies conditions, suitable in environment that should not be disturbed, avoid getting into dangerous and wise environments for frequent studies, and an economical solution to collect information in the long term.
One of the most important issues in sensor networks is routing, fault tolerance and topology control [3, 4]. For each of these issues, algorithms and protocols have been presented so far. The innermost characteristics of the wireless sensor network make it possible to differentiate from other wireless networks such as cellular and mobile networks. The routing problem in these networks is very different and challenging. Firstly, there is no possibility to create a global address scheme. Therefore, IP-based classical protocols cannot be used. Secondly, unlike telecommunication networks, in most applications of sensor networks, the flow of information is from several areas (source) to one destination node. Thirdly, traffic generated at the network level has a high redundancy. This redundancy can be realized due to generated repetitive and similar information of an event by several nodes that are in neighboring an event. Routing protocols should consider this redundancy issue especially the routing protocols that their goals are reducing power and bandwidth consumptions [5]. Fourth, the sensor nodes are severely restricted in terms of range of transmission, power range, storage capacity, and processing. Therefore, they need to have appropriate resources management. The routing protocols of the sensor networks are categorized into different classes in terms of how the environment responds. They are based on multi-paths routing, service quality, negotiation, query, and coherent protocols [6, 7]. Moreover, routing protocols are categorized into three types in terms of how they originate to the destination. They are reactive, proactive and composite. Finally, routing protocols are categorized into three groups as flat, hierarchical and location based according to operability rate of nodes, the available information for each node, and network goals.

Due to the expansion of nodes in an abnormal and uncontrollable environment and the limitations of sensor networks, these networks are prone to fail occurrence. Therefore, one of the other critical design network factor is fault tolerance. It refers to the ability of the system that handles the network despite the errors and fails in the network [8]. The ultimate goal of fault tolerance is to build reliable, quality and universally accepted systems. The reliability is the ability of a system to present and deliver services based on the desire users. So that the service can be provided and be rely. In this case, it is necessary firstly the information reaches to the destination, and secondly, the information arrived must be correct. Because, decision-making based on wrong information is much worse than non-decision. The fault tolerance in sensor networks is in fact, the ability of system to maintain the function and functionality of the sensor network without any interruption in the presence of node degradation [9].

In the section 2 of the paper is introduced an energy efficient routing protocol based on automated learning method. So, the simulation results and evaluation of it is discussed in the section 3. Finally, the conclusion of the paper is explained in the section 4 . 


\section{ENERGY EFFICIENT ROUTING PROTOCOL BASED ON AUTOMATED LEARNING METHOD (EER-AL)}

In this section, an energy efficient routing protocol based on automated learning method is proposed. Before describing this protocol, it is necessary to talk about this learning method.

\subsection{DEFINITION}

The process of learning creatures is one of the new research topics [10]. These researches are divided into two general categories. The first category recognizes the principles of learning the living entities and its stages. The second group seeks to provide a methodology for putting these principles in a machine. Learning is defined as changes made in the performance of a system based on experiences [11]. An important feature of learning systems is the ability to improve their efficiency over time. In mathematical terms, it can be stated that the purpose of a learning system is to optimize a task that is not well known [12, 13]. Therefore, an approach to this problem is to reduce the goals of the learning system to an optimization problem, which is defined on a set of parameters and aims to find a set of optimal parameters. In many of the issues raised, there is no knowledge of the correct answers to the problem, which is required by supervised supervision. For this reason, the use of a learning method called Reinforcement Learning (RL) has been considered. It has many applicative areas such as wireless sensor networks [14]. This category of learning method is an orthogonal approach to solving different and more difficult problems. It uses a combination of dynamic programming and supervisory learning to achieve a powerful machine learning system. In the RL is defined a goal for the learning agent so the agent must be achieving it. Consequently, the agent learns how to achieve the target by different tests in various environment $[14,15]$. In the $\mathrm{RL}$, a learner's factor in learning through repeated interactions with the environment leads to an optimal control policy. The effectiveness of these interactions with the environment is evaluated by the maximum (minimum) of the number of rewards (penalty) taken from the environment. The main advantage of the $\mathrm{RL}$ over other learning methods is the need for no information from the environment (except amplification signal) [11]. One of the enhancement learning methods is Stochastic Learning Automata (SLA) [16, 17]. It tries to find the answer to the problem without any information about the optimal operation. An automated operation is randomly selected and applied in the environment. Then, the received environment response and the probability of the actions are updated according to the learning algorithm and the procedure is repeated. A random automata that works in the above way to increase its efficiency is called a SLA.

A learning automaton consists of two main parts. Stochastic automata with a limited number of operations and a random environment in which the automaton is associated with it. The other is the learning algorithm that the automata learns the optimal operation using it. A random automaton is defined as $S A \equiv\{\alpha, \beta, F, G, \varphi\}$, where, $\alpha$ shows set of automata actions and $\alpha$ is defined from index one to $r$, so, $r$ is number of automatic actions $\alpha \equiv\left\{\alpha_{1}, \alpha_{2}, \ldots, \alpha_{r}\right\} . \beta$ is input sets of automata that its domain is assumed as $m$ so $\beta \equiv\left\{\beta_{1}, \beta_{2}, \ldots, \beta_{m}\right\}$. F is new status generator function that is defined as $F \equiv \varphi \times \beta \rightarrow \varphi$. $G$ is output function that the output function that maps the current state to the next output $(G \equiv \varphi \rightarrow \alpha)$. $\phi$ presents the set of internal states of the automata is at the $n$ moment as $\varphi(n) \equiv\left\{\varphi_{1}, \varphi_{2}, \ldots, \varphi_{k}\right\}$. The set $\alpha$ includes automated outputs (actions), in which the automation in each step chooses an operation of $r$ for this set to apply to the environment. If the mapping $F$ and $G$ are definite, the automata is called deterministic automata. When the $F$ and $G$ maps are random, the automata is called non-deterministic automata.

Learning automata are divided into two groups of fixed and variable structure automata. In stochastic automata with a fixed structure, the probabilities of automated operations are constant. While in stochastic automata with a variable structure, the probabilities of automated operations are updated in each repetition. In this structure, changing the likelihood of actions is done based on the learning algorithm and the internal state of the automata is represented by the probabilities of the operation of the automata. In fact, the automaton is considered state-output automata that its output is equivalent to its internal state. The action probability vector of the operation that is defined at the follow Eq.(1) defines the internal state of the automaton at the instant $n$.

$$
P(n) \equiv\left\{p_{1}(n), p_{2}(n), \ldots, p_{r}(n)\right\}
$$

So that, at the beginning of the activity of the automaton, the probability of its operation is equal and equal $(r)$ is the number of automatic operations).

$$
\sum_{i=1}^{r} p_{i}(n)=1 \forall n, p_{i}(n)=\operatorname{Prob}\left[\alpha(n)=\alpha_{i}\right]
$$

The environment can be represented as $E \equiv\{\alpha, \beta, c\}$, where $\alpha$ shows the input sets of environment, $\beta$ presents the output sets of environment, and $c$ introduces set of penalty probabilities.

$$
\left(\alpha \equiv\left\{\alpha_{1}, \alpha_{2}, \ldots, \alpha_{r}\right\}, \beta \equiv\left\{\beta_{1}, \beta_{2}, \ldots, \beta_{m}\right\}, c \equiv\left\{c_{1}, c_{2}, \ldots, c_{r}\right\}\right)
$$

The input of the environment is one of the $r$ automata actions. The output (response) of the environment to each action is specified by $\beta$. If the $\beta_{i}$ is a binary response, the environment is called the P-Model. In such an environment, $\beta_{i}(n)=1$ is as an inappropriate or failure response. The appropriate or successful answer is when the $\beta_{i}(n)$ is zero. Other environment model is QModel. In this model, $\beta_{i}(n)$ contains a limited number of values in the interval $[0,1]$. In addition, in S-Model, $\beta_{i}(n)$ is a random variable in the interval $[0,1]$. As mentioned above, $c$ specifies the probabilities of penalty (failures) of environmental responses and is defined as Eq.(3).

$$
c_{i}=\operatorname{Prob}\left\{\beta(n)=1 \mid \alpha(n)=\alpha_{i}\right\}, i=\{1,2, \ldots, r\}
$$

It shows that $\alpha_{i}$ may receive an undesirable response from the environment. The values of $\alpha_{i}$ are unspecified and is assumed the $C_{i}$ have at least one unique value. The same way, the environment can be represented by the set of reward probabilities (success). So, it is shown by $d_{i}$. The $d_{i}$ indicates the probability of receiving the desired response to the action of $\alpha_{i}$. In static environments, the probability of penalty of $\alpha_{i}$ are constant. While in non-stationary environments, the probabilities of fines change over time. The connection of random automata with the environment is shown in Fig.1. 


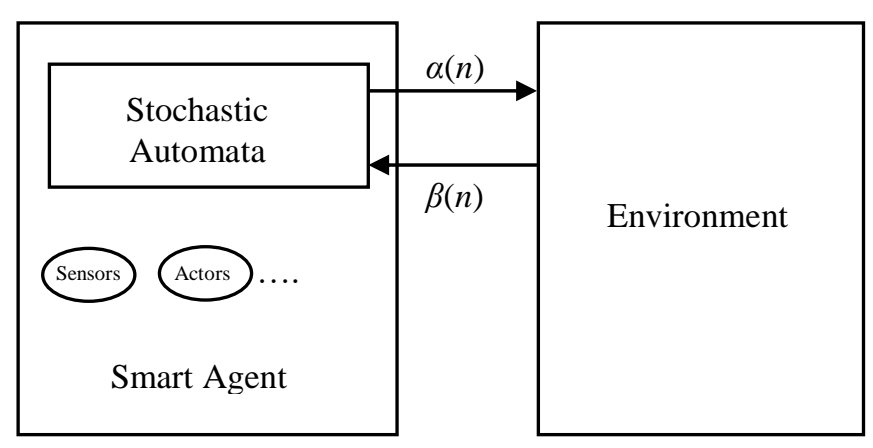

Fig.1. SLA Schema

The Fig.1 refers a learning algorithm that is called the Stochastic Learning Automata (SLA). Similarly, SLA can be defined as $L A \equiv\{\alpha, \beta, p, T\}$. Where, $\alpha$ is defined from index one to $r$, so, $r$ is number of automatic actions. $\alpha \equiv\left\{\alpha_{1}, \alpha_{2}, \ldots, \alpha_{r}\right\} . \beta$ is input sets of automata that its domain is assumed as $r$ so $\beta \equiv\left\{\beta_{1}, \beta_{2}, \ldots, \beta_{r}\right\} . p$ shows action probability vector and is represented as $p \equiv\left\{p_{1}, p_{2}, \ldots, p_{r}\right\}$. In this case, our learning algorithm is

$$
T \equiv p(n+1)=T[\alpha(n), \beta(n), p(n)]
$$

\subsection{ROUTING PROTOCOL BASED AUTOMATED LEARNING METHOD}

ON

Recent research on routing sensor networks indicates that researchers are more interested in providing energy-aware routing to increase network lifetime [2, 5, 18, 19, 20, 22]. In the network lifetime is various definitions that three of them are very important. First definition, the time interval from the beginning of the network activity until the network is converted to an unconnected network due to the completion of the energy of one or more nodes. In second definition, the time interval from the beginning of the network activity to the time when the energy of a certain percentage of nodes is completed and in last definition, the time interval from the beginning of the network activity to the time when the energy of the first node in the network ends [21]. Each of these attempts, considering the criteria, has tried to provide a proactive energy-aware protocol. Among the criteria that have been used, the shortest distance and minimum energy consumption can be mentioned. In this paper is introduced a new energy efficient routing protocol based on automated learning method that is called the EER-AL. We use the learning automata to select the appropriate routes for sending data packets, balance the energy consumption among the nodes and hence to increase the network lifetime (based on the third definition). In this protocol, the nodes create their routing tables by using the flood propagation carried out by the base station node. Whenever a packet node has to be sent, using its learning automaton, it selects a path from the possible paths and sends the packet to the base station node. If the selected path is appropriate according to the desired criteria, the action selected by the learning automata will be rewarded and hence the probability of choosing this path for the next steps will be increased. Otherwise, the action selected by the learning automata will be fined and, consequently, the probability of choosing it will be reduced. In suggested protocol is assigned a learning automaton to each node in the network. The protocol includes two phases of casting and routing and learning.
The casting phase is begun with the node that have data for transmission. It broadcast a message is called HELLO to its neighbors. This package contains three fields of the transmitter node number, the number of steps to the base station node and the transmitter node's energy level. Neighbors also send it to their neighbors by receiving this packet. Once the base station node receives the related packet, it creates a packet called Rep-HELLO and publish it on the network. The base station node initializes Rep-HELLO packet fields before the casting it. These fields are consist of sender node number, hop counter and energy level. Other nodes after receiving the Rep-HELLO packet and its associated information, adds the path to its routing table and publish the packet on the network. This phase is completed when all network nodes reach the Rep-HELLO packet. At the end of this phase, each node has several paths to the base station. Each node calculates the probability of choosing the path according to Eq.(5).

$$
P(i)=1-\frac{h_{i}}{\sum_{j=1}^{n g h} h_{j}}
$$

In the Eq.(5), $h_{i}$ is the number of hops to the base station node for the $i^{\text {th }}$ path and $n g h$ represents the number of paths in the routing table. Each node is equipped with a learning automaton whose number of operations is equal to the number of paths of that node to the base station node. In addition, the probability of choosing each action is equal to the probability of choosing the corresponding path in the routing table. In fact, the actions of learning automata have a one-to-one correspondence with the paths of the routing table.

The routing and learning phase is begun when the source node received the Rep-HELLO packet. The source node selects the path that has the maximum probability and sends data packets to this node through this path. The intermediate nodes also continue to do so until the packet reaches the base station node. Each node waits for the response from its destination nodes after sending the data packet. If the answer is positive, the desired path is rewarded.

In this protocol, routing tables include four fields of the next node number, the probability of selection, the energy of the next node and the number of hops to the base station node. The Pseudo code of the proposed protocol is shown below.

For each node (؛!)

\{

If Generate (DATA packet)

Select one Path // with the aid of Learning Automata

Transmit(DATA packet) on the selected path

Wait for ACK

If received(DATA packet)

Transmit(ACK packet) to sender node

If notDestination

Select one Path // with the aid of Learning

Automata

Transmit(DATA packet) on path

Wait for ACK

If received(ACK packet)

$\begin{aligned} \text { If } \mathrm{ACK}= & =\text { "reward" then } \\ & \text { Reward the path } \\ \text { If } \mathrm{ACK}= & =\text { "Penalty" then } \text { " } \\ & \text { Penalize the path }\end{aligned}$


\}

The updating of routing table is done based on follow steps:

- If the node receives only one HELLO packet, it will add the received packet information as a record of its routing table and the probability of choosing a path equals one.

- If a node receives more than one HELLO packet, it will enter the information of all received packets in its routing table (each record for a path). The probability of selecting each of these paths is determined by the relationship in Eq.(6).

$$
\forall i \quad i \leq m \quad P_{i}=h \frac{\frac{1}{\text { numhop }_{i}}}{\sum_{i=1}^{m} \frac{1}{\text { numhop }_{i}}}+(1-h) \frac{\text { energylevel }_{i}}{\sum_{i=1}^{m} \text { energylevel }_{i}}
$$

where, $i$ is received packet number, numhop $_{i}$ is number of hops for the $i^{\text {th }}$ packet, energylevel $i_{i}$ is the transmitter node energy of $i^{\text {th }}$ packet, $m$ is number of packets received. $h$ is a constant that is a number between zero and one. This parameter represents the ratio of the effect of the number of steps to the node energy that is selected. If this parameter is closer to one, the effect of the number of hops will be greater. In addition, the effect of energy will be greater on the initial probability of choosing a path if the value of this parameter is closer to zero. The receiver node initialized the fields of HELLO packet so, it introduces your number as the sender node and it measures its energy level in the field. To set the number of hops, one unit is added to the number of path hop, which has the lowest number of steps in its routing table, and places it in the desired field.

Each node, according to its routing table, creates a learning automaton whose number of operations is equal to the number of paths in the routing table. In fact, there is a one-to-one correspondence between the actions of the learning automaton and the routing table paths. The probability of choosing each action is equal to the probability of the corresponding path in the table. When the learning automata selects a practical node, the path corresponding to that action is selected to send the desired packet to the base station node. If the selected path is appropriate, the chances of choosing it will be increased according to the learning algorithm. Otherwise, the choosing probability will be decreased. At the end of this phase, each node has an automated learning method and a routing table, which will be used them to direct the data packet to the base station node. Each node that receives a data packet, if its destination is not closed, directs it to the base station node using its automated learning method and routing table. Energy management of proposed protocol is rely on Eq.(7).

$$
V_{p}=\left(1+\frac{\frac{\text { energylevel }_{i}}{\text { avgenergy }}-0.5}{0.3}\right) \beta
$$

where, avgenergy is average energy of the first nodes of other paths, energylevel $i$ is the energy level of a node that ACK packets come from it. In addition, $\beta$ is determined by the Eq.(8). The value of 0.3 is the difference of $50 \%$ of $80 \%$ between $\beta$ and $2 \beta$.

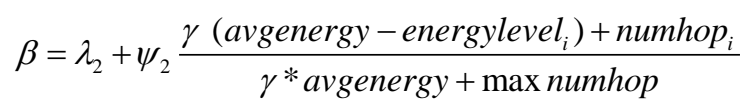

In addition, we define the reward parameter that is called $\alpha$. It is calculated from Eq.(9).

$$
\alpha=\lambda_{1}+\psi_{1} \frac{\gamma^{*} \text { energylevel }_{i}+\left(\max \text { numhop }- \text { numhop }_{i}\right)}{\gamma * \text { energylevel }^{2} \max \text { numhop }}
$$

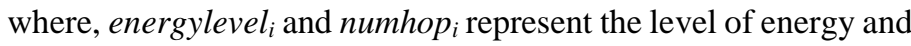
the number of nodes that ACK packets come from it. energylevel is initial energy of the desired node. The maxnumhop represents the maximum number of hops from the node receiving the ACK packet to the base station node. $\lambda_{1}$ and $\lambda_{2}$ represent the minimum acceptable value for reward and penalty parameters, respectively. The $\gamma$ is different scale between numhop and energylevel and it should be chosen in such a way that these two semesters be on an almost equal scale. The $\psi_{1}$ and $\psi_{2}$ are chosen so that the values of $\alpha$ and $\beta$ parameters do not exceed the specified limit.

\section{SIMULATIONS}

To simulate the network environment, MATLAB software is used. The energy model used in these simulations is the energy model as described in [23]. In learning algorithms, the parameters $\lambda_{1}$ and $\lambda_{2}$ are equal to 0.1 ; the parameters $\psi_{1}$ and $\psi_{2}$ are equal to 0.15 and the value of parameter $p$ is 3 . The location of the nodes in the network is determined randomly. The simulations have done in four scenarios. One of them is in the $1000 \mathrm{~m} \times 1000 \mathrm{~m}$ environment with 100, 200, 300 and 400 nodes. The objective of the sensor network is to collect temperature changes in the agriculture lands. The sensor nodes sense the temperature changes and transmit the relevant information to the base station node. The transmission range of each node is assumed 250 meters. In the simulation environment, the proposed protocols are compared with the TinyLAP [24], EAR [25], and PGR [26] protocols. This comparison is based on the initial parameter that are seen in Table.1. In addition, the output parameters of the number of control packets sent and received at the beginning and the end of the network life. The results of simulation are presented in Fig.2 and Fig.4. In addition, the energy saving rates of each protocol are compared together and their results are shown in Fig.4.

Table 1. Values of input parameters for EER-AL

\begin{tabular}{|c|c|}
\hline Parameter & Value \\
\hline Initial (max) energy & $1 \mathrm{~J} / \mathrm{bit}$ \\
\hline Radio/ Sensor energy consumption & $40 \mathrm{~nJ} / \mathrm{bit}$ \\
\hline Transmit process cost & $40 \mathrm{~nJ} / \mathrm{bit}$ \\
\hline Receive/sense process cost & $10 \mathrm{~nJ} / \mathrm{bit}$ \\
\hline Data packet size & $500 \mathrm{bytes}$ \\
\hline Sensing Radius & $6 \mathrm{~m}$ \\
\hline Receive buffer size & $1000 \mathrm{bytes}$ \\
\hline Send buffer size & $1000 \mathrm{bytes}$ \\
\hline Deployment area size & $(1000 \times 1000) \mathrm{m}^{2}$ \\
\hline Send/receive buffer counts & 20 \\
\hline Sink position & $(310 \times 310) \mathrm{m}^{2}$ \\
\hline Transmission Radius & $10 \mathrm{~m}$ \\
\hline
\end{tabular}

In the Fig.2, the number of control packets in the early days of the network (receiving around 50,000 data packets by the base 
station node of the resources) for the EER-AL protocol is lower than the other protocols. Because, the proposed protocol only needs the initial release of the HELLO packet.

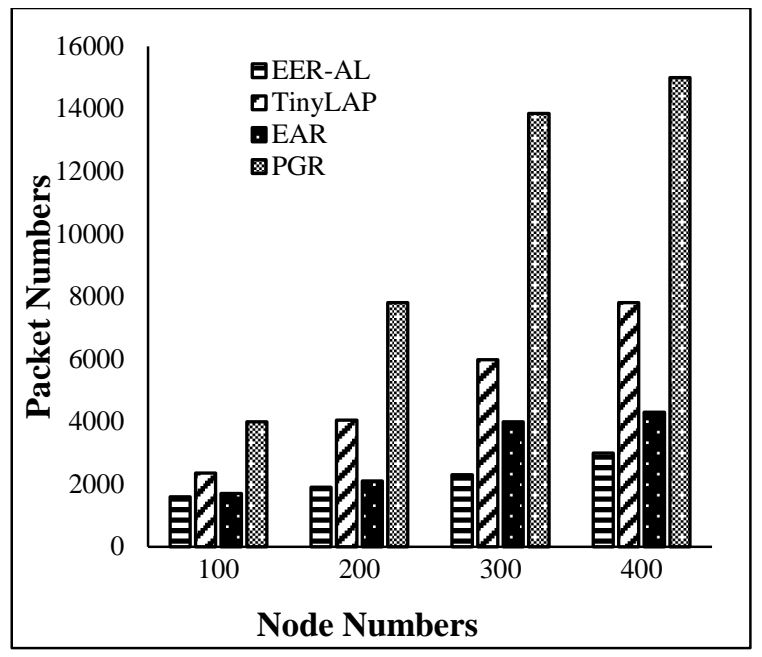

Fig.2. Number of control packets received and sent at the beginning of network operation for EER-AL, TinyLAP, EAR and PGR protocols

In the Fig.3, the number of control packets of the four protocols examined in the late-life of the network is seen in different environments. The number of received and sent control packets in the late life of the network for the TinyLAP protocol is greatly increased. Because, the warn packets, which are used in the TinyLAP protocol, are increased quickly. This number will be higher in the EAR protocol due to the repetition of the overall release phase at the end of the network lifetime than the network's early work. There is no change for the two EER-AL and PGR protocols, because sending and receiving control packets in these two protocols only takes place in the very early days of the network.

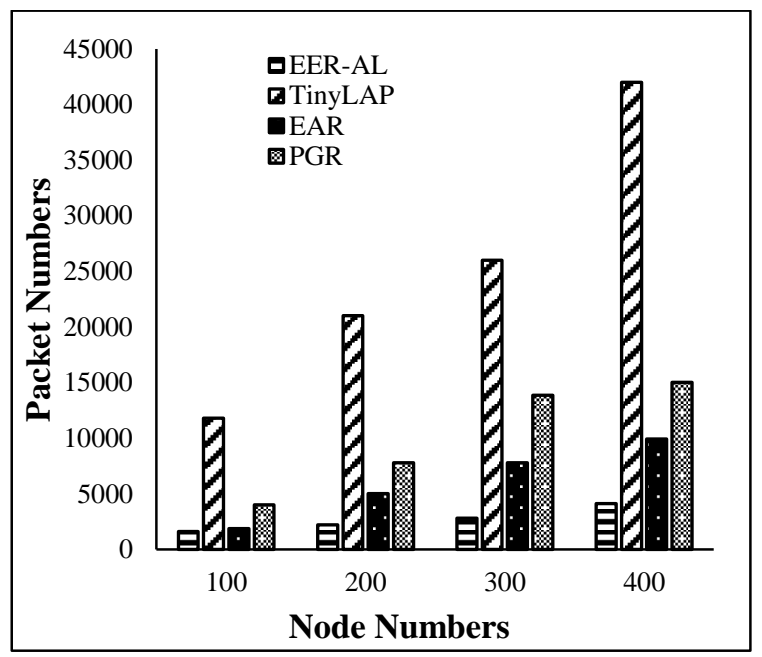

Fig.3. The number of receptions and sent control packets in the late life of the network for EER-AL, TinyLAP, EAR and PGR protocols

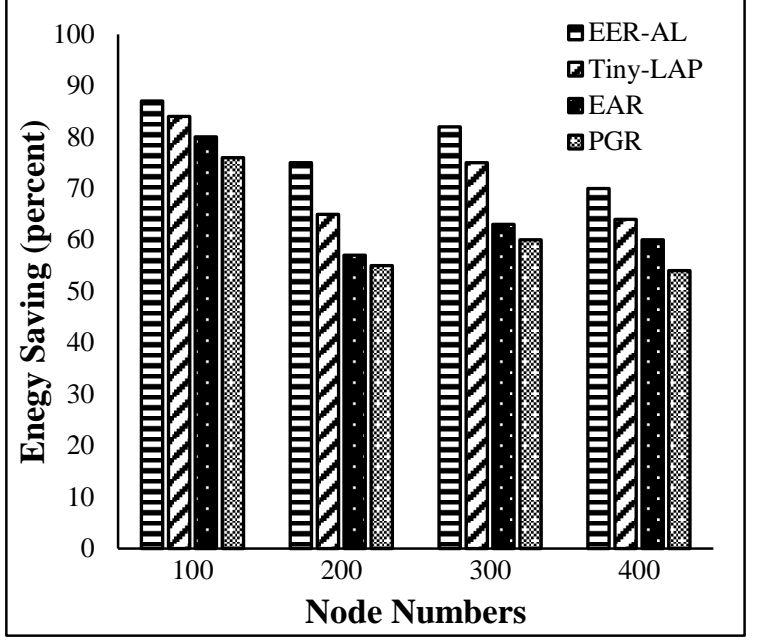

Fig.4. Energy saving rate in the EER-AL, TinyLAP, EAR and PGR protocols

\section{CONCLUSIONS}

In this paper, the use of learning automata for routing in sensor networks was proposed. In this protocol, the nodes select the data paths so that the energy of the nodes is consumed as a balance using the automated learning method. As a result, the network lifetime is increased. The simulation results and the comparison of this protocol with other protocols indicate that this protocol has best performance. Unlike the EAR and PGR protocols, the proposed protocol does not require location information.

\section{REFERENCES}

[1] Z. Mohammed and E. Ahmed, "Internet of Things Applications, Challenges and Related Future Technologies", World Scientific News, Vol. 67, No. 2, pp. 126-148, 2017.

[2] F. Kiani, "AR-RBFS: Aware-Routing Protocol Based on Recursive Best-First Search Algorithm for Wireless Sensor Networks", Journal of Sensors, Vol. 2016, pp. 1-10, 2016.

[3] L.B. Bhajantri and N. Nalini, "A Fault Tolerance Approach to Topology Control in Distributed Sensor Networks", Proceedings of IEEE International Conference on Advanced Communication Control and Computing Technologies, pp. 208-212, 2012.

[4] H. Bagci, I. Korpeoglu and A. Yazici, "A Distributed FaultTolerant Topology Control Algorithm for Heterogeneous Wireless Sensor Networks", IEEE Transactions on Parallel and Distributed Systems, Vol. 26, No. 4, pp. 914-923, 2015.

[5] F. Kiani, "Maximizing Wireless Sensor Network Lifetime Based on Linear Programming Method", International Research Journal of Engineering and Technology, Vol. 3, No. 3, pp. 1354-1359, 2018.

[6] J.N. Al-Karaki and A.E. Kamal, "Routing Techniques in Wireless Sensor Networks: A Survey", Proceedings of the IEEE International Conference on Wireless Communications, pp. 6-28, 2004.

[7] A. Sarkar and T. Murugan, "Routing Protocols for Wireless Sensor Networks: What the Literature Says?", Alexandria Engineering Journal, Vol. 55, No. 4, pp. 3173-3183, 2016. 
[8] A. Avizienis, J.C. Laprie and B. Randell, "Fundamental concepts of dependability", Proceedings of the Technical report, UCLA CSD Report no. 010028, 2001.

[9] F. Koushanfar, M. Potkonjak and A. Sangiovanni, "FaultTolerance Techniques for Sensor Networks", Proceedings of IEEE International Conference on Sensors, pp. 14911496, 2002.

[10] V. Saritha, P. V. Krishna, S. Misra and M.S. Obaidat, "Learning Automata based Optimized Multipath routing using Leapfrog Algorithm for VANETs", Proceedings of International Conference on Mobile and Wireless Networking, pp. 1-5, 2017.

[11] K.S. Narendra and M.A.L. Thathachar, "Learning Automata: An Introduction", Prentice Hall, 1989.

[12] Z. Shariat, A. Movaghar and, M. Hoseinzadeh, "A Learning Automata and Clustering-based Routing Protocol for Named Data Networking", Telecommunication System, Vol. 65, No. 1, pp. 9-29, 2017.

[13] H. Ge and Sh. Li, "A Parameter-Free Learning Automaton Scheme", Available at: https://pdfs.semanticscholar.org/4499/e181afb78f0ce8043a ccf2db37c3a90314c0.pdf

[14] F. Kiani, "Reinforcement Learning Based Routing Protocol for Wireless Body Sensor Networks", Proceedings of IEEE 7th International Symposium on Cloud and Service Computing, pp. 71-78, 2017.

[15] K. Arulkuraman, M. Peter, M. Brundage and A. Bharath, "Deep Reinforcement Learning: A Brief Survey", IEEE Signal Processing Magazine, Vol. 5, No. 3, pp. 26-38, 2017.

[16] C. S. Chasparis, "Stochastic Stability of Perturbed Learning Automata in Positive-Utility Games", Available at: https://arxiv.org/pdf/1709.05859.pdf

[17] G. Barto and P. Anandan, "Pattern-Recognizing Stochastic Learning Automata", IEEE Transactions on Systems, Man, and Cybernetics, Vol. 15, No. 3, pp. 360-375, 1985.
[18] S. Jabbar et al., "Analysis of Factors Affecting Energy Aware Routing in Wireless Sensor Network", Wireless Communications and Mobile Computing, Vol. 2018, pp. 121, 2018.

[19] K. Arasu and R. Ganesan, "Effective Implementation of Energy Aware Routing for Wireless Sensor Network", Materials Today: Proceedings, Vol. 5, No. 1, pp. 11861193, 2018.

[20] S. Aswale and V.R. Ghorbade, "LQEAR: Link Quality and Energy-Aware Routing for Wireless Multimedia Sensor Networks", Wireless Personal Communications, Vol. 97, No. 1, pp. 1291-1304, 2017.

[21] M. Ilyas and I. Mahgoub, "Handbook of Sensor Networks: Compact Wireless and Wired Sensing Systems", CRC Press, 2005.

[22] H. Zhoue et al., “A Multiple-Dimensional Tree Routing Protocol for Multi sink Wireless Sensor Networks based on Ant Colony Optimization", International Journal of Distributed Sensor Networks, Vol. 2012, pp. 1-10, 2012.

[23] C. Intanagonwiwat et al., "Directed Diffusion for Wireless Sensor Networking", IEEE/ACM Proceedings of the Transactions on Networking, Vol. 11, No. 1, pp. 2-16, 2003.

[24] M. Ankit et al., "TinyLAP: A Scalable Learning AutomataBased Energy Aware Routing Protocol for Sensor Networks", Proceedings of International Conference on Wireless and Communications, pp. 1-8, 2006.

[25] D. De, W. Song and Sh. Tang, "EAR: An Energy and Activity-Aware Routing Protocol for Wireless Sensor Networks in Smart Environments", The Computer Journal, Vol. 55, No. 12, pp. 1492-1506, 2012.

[26] T. Roosta, "Probabilistic Geographic Routing protocol for Ad Hoc and Sensor Networks", Proceedings of International Workshop Wireless Ad Hoc Networks, pp. 1-8, 2006. 